\title{
An adaptive study to determine the optimal dose of the tablet formulation of the PARP inhibitor olaparib
}

J Mateo, ${ }^{1}$ V Moreno, ${ }^{1}$ A Gupta, ${ }^{2}$ SB Kaye,${ }^{1}$ E Dean, ${ }^{3}$ MR Middleton, ${ }^{2}$ M Friedlander,${ }^{4} \mathrm{C}$ Gourley, ${ }^{5}$ R Plummer, ${ }^{6}$ G Rustin, ${ }^{7}$ C Sessa, ${ }^{8}$ K Leunen, ${ }^{9}$ J Ledermann, ${ }^{10} \mathrm{H}$ Swaisland, ${ }^{11}$ A Fielding, ${ }^{11}$ W Bannister, ${ }^{12}$ S Nicum, ${ }^{2}$ LR Molife ${ }^{1 *}$

${ }^{1}$ Drug Development Unit, The Royal Marsden/The Institute of Cancer Research, Sutton, UK; ${ }^{2}$ Department of Oncology, University of Oxford, Oxford, UK; ${ }^{3}$ Clinical Trials Unit, The Christie NHS Foundation Trust/University of Manchester, Manchester, UK; ${ }^{4}$ Prince of Wales Clinical School, University of New South Wales, Prince of Wales Hospital, Randwick, Australia; ${ }^{5}$ University of Edinburgh Cancer Research UK Centre, MRC IGMM, Edinburgh, UK; ${ }^{6}$ Northern Centre for Cancer Care, Newcastle-upon-Tyne, UK; ${ }^{7}$ Mount Vernon Hospital, Northwood, UK; ${ }^{8}$ Oncology Institute of Southern Switzerland, Bellinzona, Switzerland; ${ }^{9}$ Department of Obstetrics and Gynecology, Division of Gynecologic Oncology, University Hospitals Leuven, Leuven, Belgium; ${ }^{10}$ UCL Hospitals and UCL Cancer Institute, London, UK; ${ }^{11}$ AstraZeneca, Macclesfield, UK; ${ }^{12}$ PHASTAR, London, UK

*Correspondence: $\operatorname{Dr}$ L Rhoda Molife MD, Drug Development Unit, The Royal Marsden/The Institute of Cancer Research, Downs Road, Sutton SM2 5PT, UK. Tel: +44 208915 6142. Fax: +44 208642 7979. Email: maria.bynoe@icr.ac.uk

Word count: 4231

Figures/tables: 1/6

References: 25

Running heading: Adaptive study to determine olaparib tablet dose 
Acknowledgements: The authors would like to thank the patients who took part in the study, as well as Wendy Burke and Peter McCormack for their assistance with the PK analysis. The following clinical centres acknowledge infrastructure funding from the Experimental Cancer Medicine Centre initiative for early-phase clinical trials: The Royal Marsden/The Institute of Cancer Research, The Christie NHS Foundation Trust, The Oxford Cancer and Haematology Centre, Edinburgh Cancer Research Centre, The Northern Institute for Cancer Research and University College London.

This study was sponsored by AstraZeneca. Medical writing assistance was provided by Claire Routley PhD from Mudskipper Business Ltd, funded by AstraZeneca. 


\section{ABSTRACT}

Background: Olaparib is poorly soluble, requiring advanced drug delivery technologies for adequate bioavailability. Sixteen capsules/day are required for the approved $400 \mathrm{mg}$ twice daily dose; a tablet formulation was developed to reduce pill burden. This clinical trial evaluated the optimal dose and administration schedule of the tablet formulation.

Patients and methods: Two stages of sequentially enrolled cohorts: stage 1, pharmacokinetic properties of tablet and capsule formulations were compared in patients with advanced solid tumours; stage 2, tablet dose escalation with expansion cohorts at doses/schedules of interest in patients with solid tumours and BRCAm breast/ovarian cancers.

Results: Olaparib $200 \mathrm{mg}$ tablet displayed similar $\mathrm{C}_{\text {max,ss }}$ but lower $\mathrm{AUC}_{\mathrm{ss}}$ and $\mathrm{C}_{\mathrm{min}, \mathrm{ss}}$ than $400 \mathrm{mg}$ capsule. Following multiple dosing, steady-state exposure with tablet $\geq 300$ $\mathrm{mg}$ matched or exceeded that of $400 \mathrm{mg}$ capsule. After dose escalation, while $400 \mathrm{mg}$ twice daily was the tablet maximum tolerated dose based on haematological toxicity, $65 \%$ of patients in the randomized expansion phase eventually required dose reduction to $300 \mathrm{mg}$. Intermittent tablet administration did not significantly improve tolerability. Tumour shrinkage was similar for 300 and $400 \mathrm{mg}$ tablet and $400 \mathrm{mg}$ capsule cohorts.

Conclusions: The recommended monotherapy dose of olaparib tablet for Phase III trials was $300 \mathrm{mg}$ twice daily, simplifying drug administration from 16 capsules to four tablets per day.

Clinical trial number: NCT00777582 (ClinicalTrials.gov)

Word count: 218 (max 250) 


\section{KEY POINTS}

- An alternative tablet formulation of the PARP inhibitor olaparib with improved bioavailability has been developed to facilitate olaparib administration to patients.

- Following multiple dosing, steady-state exposure with olaparib tablet $\geq 300 \mathrm{mg}$ matched or exceeded that of the olaparib $400 \mathrm{mg}$ capsule.

- Efficacy in relation to tumour shrinkage was similar between olaparib 300 and 400 mg tablet and 400 mg capsule doses; owing to haematological toxicity, tolerability was improved with olaparib $300 \mathrm{mg} \mathrm{BD}$ tablet formulation.

- The recommended monotherapy dose of olaparib tablet for Phase III trials was 300 mg twice daily, simplifying drug administration from 16 capsules to four tablets per day. 


\section{INTRODUCTION}

Poly(ADP-ribose) polymerases (PARPs) play a key role in detecting DNA damage and triggering activation of DNA repair pathways. Two seminal publications laid the foundation for clinical development of PARP inhibitors in the treatment of cancers harbouring a $B R C A 1 / 2$ mutation $[1,2]$. Individuals carrying $B R C A$ mutations $(B R C A m)$ account for $15 \%$ of cases of high-grade serous ovarian carcinoma $[3,4]$ and $10-20 \%$ of cases of triple-negative breast cancer (oestrogen-receptor-, progesterone-receptor-, and HER2-negative tumours) [5, 6].

A proof-of-concept clinical trial of olaparib (Lynparza ${ }^{\mathrm{TM}}$ ), a potent oral inhibitor of PARP1 and PARP2, established $400 \mathrm{mg}$ capsule (CAP) formulation twice daily (BD) as the maximum tolerated dose (MTD) [7]. Drug exposure increased proportionally with doses up to $100 \mathrm{mg} \mathrm{BD}$, with less-marked increases above this threshold, and pharmacodynamic (PD) effects plateaued at doses $>60 \mathrm{mg} \mathrm{BD}$. However, further clinical trials in breast and ovarian BRCAm cancer suggested a dose-response relationship, supporting use of the MTD of olaparib over a minimal biologically effective dose [8-10].

Subsequent Phase II studies of olaparib confirmed the sustained antitumour activity in patients with germline $B R C A m(g B R C A m)$ high-grade serous epithelial ovarian $[8,10]$, breast and other tumour types $[9,11]$; olaparib antitumour activity was also observed in sporadic cancers [12]. 'BRCAness' is used to refer to tumours with BRCA-like genomic or post-transcriptional aberrations, suggesting a wider application of PARP inhibitors [1315] beyond tumours associated with BRCAm. In a randomized Phase II study in patients with platinum-sensitive recurrent ovarian cancer, olaparib maintenance therapy improved progression-free survival (PFS) and patients with BRCAm were most likely to benefit from treatment $[16,17]$. In December 2014, the CAP formulation of olaparib received EU [18] and US [19] approval. To receive the approved $400 \mathrm{mg}$ BD CAP dose, 
patients need to take $8 \times 50 \mathrm{mg}$ large size 0 capsules twice daily. To improve dosing constraints of the CAP formulation, a melt-extrusion tablet (TAB) formulation with improved bioavailability was developed.

We report results of an open-label, multicentre, multistage, Phase I trial comparing the pharmacokinetics (PK), efficacy and tolerability of different doses and scheduling of the olaparib CAP and TAB formulations to determine an optimal TAB dosing strategy for Phase III studies of olaparib. 


\section{PATIENTS AND METHODS}

\subsection{Study design}

The Phase I study was conducted in two sequential stages (Online Resource 1, Supplementary Figure 1; ClinicalTrials.gov NCT00777582): stage 1 was a randomized, two-period, crossover design to determine the comparative bioavailability of the two formulations (bioavailability assessment; patients could then enter a continued-supply phase [CSP; cohorts 1-3]) followed by a dose-expansion phase (groups 1-2) to validate PK modelling and determine steady-state PK; stage 2 determined the MTD and optimum schedule of the TAB formulation (using dose-escalation [groups 3-5.2] and doseexpansion, randomized TAB/CAP comparison [group 6]; dose-expansion, randomized TAB alternative dosing schedules, group 8).

Institutional review boards/independent ethics committees of the 10 investigational sites approved the protocol. All patients provided written informed consent. The study was performed in accordance with the Declaration of Helsinki, Good Clinical Practice and the AstraZeneca policy on bioethics [20].

\subsubsection{Stage 1 - bioavailability assessment}

Patients were randomized to two treatment periods of olaparib CAP and TAB, separated by a 6- to 14-day washout, to study the PK profile at different dose levels (Online Resource 1, Supplementary Figure 1). On completion, patients could enter a CSP, where they received olaparib $400 \mathrm{mg}$ BD CAP until disease progression, unacceptable toxicity or withdrawal of consent. Based on an estimate of intra-subject variability following CAP dosing, a sample size of six patients for each dose level was expected to produce a $90 \%$ confidence interval $(\mathrm{Cl})$ of $1.61-2.49$ for the comparative bioavailability assessment. PK modelling and simulation was performed to select a TAB dose expected 
to have comparable steady-state maximum plasma concentration $\left(\mathrm{C}_{\max }\right)$ and area under the plasma concentration-time curve (AUC) to the $400 \mathrm{mg}$ BD CAP dose.

Once the TAB dose was determined, a dose-expansion phase was initiated to validate PK modelling for the selected TAB and $400 \mathrm{mg}$ BD CAP (group 1). A second group of six patients (group 2) was randomly assigned to one of two treatment sequences: selected TAB dose BD days 1-8 followed by $400 \mathrm{mg}$ BD CAP days 9-15 or vice versa. After day 15, all patients received selected TAB until treatment discontinuation.

Primary objectives of stage 1 were to determine comparative bioavailability of the TAB and CAP formulations and to assess safety and tolerability (group 1). Secondary objectives were to: generate single-dose PK data and information on dose linearity of the $T A B$ formulation; assess preliminary efficacy of the $T A B$ and $C A P$ formulations (group 1); compare steady-state exposure achieved with selected TAB and $400 \mathrm{mg}$ BD CAP (group 2).

\subsubsection{Stage 2 - dose-schedule optimization}

Stage 1 data indicated that higher TAB doses should be explored. The protocol was amended to include a second stage with a rolling dose-escalation design (groups 3-5.2). The MTD was defined as the maximum dose at which $\geq 33 \%$ of patients experienced dose-limiting toxicities (DLTs).

Following completion of dose escalation, a randomized safety and efficacy comparison between the selected TAB dose levels and the original CAP regimen was conducted in patients with gBRCAm breast or ovarian cancer (group 6).

To assess tolerability and scheduling of the higher daily doses, the protocol was amended to recruit patients with $g B R C A m$ ovarian cancer in a further randomized group using alternative TAB schedules (group 8). Patients were randomized 1:1:1:1 to: 
schedule A, $200 \mathrm{mg}$ three times daily (TID); schedule B, $250 \mathrm{mg}$ TID 2 weeks on, 1 week off; schedule C, 400 mg BD 1 week on, 1 week off; or schedule D, 400 mg once daily (QD).

Primary objectives of stage 2 were to: determine safety and tolerability profile of doses higher than 200 mg BD TAB (groups 3-5.2); compare safety of higher TAB doses with $400 \mathrm{mg}$ CAP dose in gBRCAm breast or ovarian cancer patients (group 6); and determine safety and tolerability of alternative TAB schedules in $\mathrm{gBRCAm}$ breast or ovarian cancer patients (group 8). Secondary objectives were to: determine single-dose and steady-state exposures with higher doses and selected schedules of TAB (groups 3-5, 8); compare single-dose and steady-state exposures of TAB with $400 \mathrm{mg}$ CAP; and assess efficacy in patients treated with TAB or CAP (groups 6,8 ).

\subsection{Patients}

Key patient eligibility criteria were: age $\geq 18$ years; advanced solid tumours with no effective treatment available; Eastern Cooperative Oncology Group (ECOG) performance status (PS) 0-2; adequate bone marrow (haemoglobin $\geq 9.0 \mathrm{~g} / \mathrm{dL}$, platelet count $\geq 100 \times 10^{9} / \mathrm{L}$, white blood cells $>3 \times 10^{9} / \mathrm{L}$ ), renal (serum creatinine $\leq 1.5 \times$ upper limit of normal [ULN]), and hepatic (total bilirubin $\leq 1.5 \times U L N$; aspartate transaminase $[\mathrm{AST}] /$ alanine transaminase $[\mathrm{ALT}] \leq 2.5 \times \mathrm{ULN}$, unless liver metastases present, in which case $\leq 5 x U L N)$ function; eligibility criteria are detailed in Online Resource 1, Supplementary Table 1. After protocol amendments, group 8 required ECOG PS 0-1 and baseline haemoglobin $\geq 10 \mathrm{~g} / \mathrm{dL}$, independent of blood transfusion, within 4 weeks of randomization.

Only patients with gBRCAm breast or ovarian cancer were recruited into groups 1 and 6 . Group 8 was limited to $g B R C A m$ patients with ovarian, primary peritoneal or fallopian tube cancer. These groups were required to have at least one measurable lesion. 


\subsection{Study endpoints and assessments}

Assessments included medical review, physical examination, vital signs, and clinical laboratory tests and were performed weekly during the first 4 weeks and 4-weekly thereafter. Adverse events (AEs) were graded according to Common Terminology Criteria for Adverse Events (CTCAE) v3.0. DLT was defined as any of the following AEs occurring in the first 28 days of treatment and judged by the investigators to be related to olaparib: grade $(G) 4$ neutropenia lasting $>5$ days or associated with fever; $G 4$ thrombocytopenia; $G \geq 2$ cardiac or neurological toxicity; any $A E$ that delayed the start of course 2 for $>14$ days from the planned date; or other G3-4 events, except fatigue, nausea and vomiting, diarrhoea, myalgia or arthralgia, unless appropriate prophylactic/therapeutic measures have been administered. Guidelines for management of common toxicities were implemented at investigational sites, including uniform rules for dose modifications. Radiological assessments were performed every 8 weeks according to Response Criteria in Solid Tumors (RECIST) v1.0. Tumour shrinkage, objective response rate (ORR) and response rate in ovarian cancer were based on CA125 (GCIG) and RECIST [21, 22].

\subsection{Pharmacokinetics and modelling analysis}

Blood samples were collected at pre-specified times during stages 1 and 2 of the study. Primary outcome variables to determine the comparative bioavailability of the TAB and CAP formulations were single-dose $C_{\max }, A \cup C$ and $A \cup C$ to time $t\left(A \cup C_{0-t}\right)$. Single-dose PK parameters of time to maximum plasma concentration $\left(t_{\max }\right)$, oral clearance $(\mathrm{CL} / \mathrm{F})$, terminal half-life $(\mathrm{t} / 2)$ and volume of distribution $\left(\mathrm{V}_{\text {area }} / \mathrm{F}\right)$ were also determined. Multipledose PK parameters for groups 1, 2 and 3-8 of the dose-escalation phase and the randomized TAB formulations included assessment of $A \cup C_{s s}, C_{m a x, s s}$ and $C_{\text {min,ss. }}$. 
PK parameters for the comparative bioavailability phase and group 2 CAP/TAB crossover phase were generated using standard non-compartmental analysis. PK data from the bioavailability assessment cohorts were used to build a population PK model for olaparib by fitting a two-compartment model with first-order absorption, first-order elimination and a lag time to the data. The population parameter and inter-individual variability estimates obtained from this model were used to predict the TAB dose, with expected steady-state plasma exposures within the range of those observed following dosing of the $400 \mathrm{mg} \mathrm{BD} \mathrm{CAP}$ dose by simulating multiple-dose PK parameters ( $\mathrm{C}_{\text {max,ss, }}$, $A U C_{s s}$ ) following administration of the TAB formulation at 1-250 mg BD doses for a patient population of 10,000 . The $C_{\max , s s}$ and $A_{U} C_{0-12, s s}$ values obtained were compared with previously determined ranges following $400 \mathrm{mg}$ BD CAP dosing, and the percentage of patients with values within these ranges was determined for each TAB dose. The chosen TAB dose for groups 1 and 2 was predicted to provide a $C_{\max , s s}$ and $\mathrm{AUC}_{\text {ss }}$ within the $400 \mathrm{mg}$ CAP range for these parameters in $95 \%$ of patients. In group 1 and in stage 2 (groups 3-8), $C_{\text {max,ss }}, C_{\text {min,ss }}$ and $A \cup C_{s s}$ at different doses and schedules of TAB were determined following sparse PK sampling and analysed using the same population PK model.

\subsection{Pharmacodynamics}

Methodology and results of the PD analysis are provided in Online Resource 1, Supplementary Table 2.

\subsection{Statistical analysis}

The extent of exposure, AEs, DLTs and percentage inhibition of PARP in peripheral blood mononuclear cells were summarized with descriptive statistics. Single-dose PK, linearity, and comparative bioavailability were assessed in stage 1. 
In stage 1, plasma concentration data and PK and PD parameters were summarized descriptively by dose level and formulation administered. Within each cohort, comparative bioavailability and $90 \%$ Cls were estimated using analysis of variance with factors for patient, formulation and period.

A planned comparison of the preliminary data on antitumour activity in patients with ovarian cancer in groups 1, 6 and 8 was conducted, based on analysis of covariance (ANCOVA) of the percentage change in tumour size after 8 and 16 weeks of treatment, using comparator data from all ovarian cancer patients receiving the CAP formulation. The ANCOVA models were adjusted for baseline tumour size and, additionally for group 8, prior platinum chemotherapy status (with categories for resistant, sensitive and no prior platinum use/unknown) [23], number of prior chemotherapy regimens (discrete variable) and peritoneal involvement at baseline (as a dichotomous variable); the latter two variables were identified as possible prognostic factors based on exploratory analysis of data from the olaparib clinical programme.

Ruling out a $20 \%$ difference (based on $<20 \%$ one-sided $80 \%$ upper confidence limit [UCL]) was used as a definition of non-inferiority for a given TAB dose compared with the $400 \mathrm{mg}$ BD CAP formulation. This difference was based on Phase II studies comparing 100 and $400 \mathrm{mg}$ CAP-based doses [8, 9]. 


\section{RESULTS}

\subsection{Patient characteristics}

Between 2008 and 2012, 210 patients (87\% female) enrolled in the study and 196 received olaparib. Fifty-one patients enrolled in stage 1 and 159 in stage 2, including 31 in the dose-escalation phase. Patient characteristics are detailed in Table 1; all patients had metastatic or locally advanced non-resectable disease at enrolment.

\subsubsection{Stage 1}

Ovarian, breast and colorectal cancers were the most common tumour types (Table 1). Median time on treatment was 87 days for patients in the PK phase (PKP)/CSP (cohorts $1-3)$.

\subsubsection{Stage 2}

Patients in the dose-escalation (groups $3-5.2 ; n=31$ ) and dose-expansion phase of stage 2 (groups 6 and $8 ; n=115)$ had ovarian $(n=114)$ or breast $(n=27)$ cancer, with the exception of five patients ( $n=1$ each; metastatic castration-resistant prostate cancer, gastric cancer, uterine leiomyosarcoma, renal cell carcinoma, oesophageal-gastric junction carcinoma). Sixty-two gBRCAm patients with ovarian, primary peritoneal or fallopian tube cancer were randomized to receive the four alternative TAB schedules (group 8). Patients in groups 6 and 8 had confirmed gBRCAm; gBRCAm status for groups 1, 6 and 8 are reported in Online Resource 1, Supplementary Table 3.

Overall, 137 patients with serous (all grades) ovarian carcinoma, including primary peritoneal and fallopian tube adenocarcinoma, received olaparib. Platinum sensitivity status was available for patients in groups 6 and 8 , of which 40/100 patients (40\%) had platinum-sensitive disease, assessed by the investigator, at study entry. The median 
number of prior lines of chemotherapy ranged from two to six across cohorts and groups.

\subsection{Bioavailability assessment}

Following PK analysis for single doses of 25 and $50 \mathrm{mg} \mathrm{TAB}$ in cohorts 1 and 2, a 250 mg dose was selected for cohort 3 , assuming dose proportionality would occur at TAB doses $>50 \mathrm{mg}$ and exposures would not exceed those previously achieved following $\leq 600 \mathrm{mg}$ CAP [7].

In all three cohorts (1-3; Figure 1 and Online Resource 1, Supplementary Table 4), absorption was rapid, with maximum concentrations observed $0.5-2$ hours after TAB dosing (compared with 1-3 hours for CAP [50, 100 and $400 \mathrm{mg}$ doses]). Following this peak, plasma concentration-time profiles declined biphasically, with a $t_{1 / 2}$ of $5-9$ hours and no change in $t_{1 / 2}$ with increasing dose. The overall mean $t_{1 / 2}$ for TAB was 6.97 hours (standard deviation [SD] 1.06 hours). The mean (SD) CL/F and $\mathrm{V}_{\text {area }} / \mathrm{F}$ was 5.42 (2.60) L/h and 54.9 (30.1) L, respectively. Exposure increased approximately dose proportionally, with geometric mean (gmean) $\mathrm{C}_{\max }$ increasing 1.9- and 7.5-fold, and gmean AUC increasing 1.6- and 12.2-fold, respectively, for a two- and 10-fold dose increase.

The relative bioavailability of TAB doses compared with CAP was higher based on $\mathrm{C}_{\max }$; however, AUC values were similar at the two lower TAB doses (Online Resource 1, Supplementary Table 4). Exposure following CAP dosing increased less than proportionally at doses $>100 \mathrm{mg}$, and both $\mathrm{C}_{\max }$ and $\mathrm{AUC}$ values were higher with 250 mg TAB than with the comparative CAP dose $(400 \mathrm{mg})$. Based on geometric least squares mean (gLSmean) $\mathrm{C}_{\max }$ and $\mathrm{AUC}$ ratios and $90 \% \mathrm{Cls}$ from the 18 patients in these three cohorts, the TAB and CAP formulations cannot be considered bioequivalent. 
Further PK modelling and simulation of data predicted that a $200 \mathrm{mg}$ BD TAB dose would deliver steady-state $\mathrm{C}_{\max }$ and $\mathrm{AUC}_{0-12}$ values within the range observed for patients receiving $400 \mathrm{mg} \mathrm{BD} \mathrm{CAP}$. To validate these modelling results, six additional patients received $200 \mathrm{mg}$ TAB and $400 \mathrm{mg}$ CAP in a crossover design (group 2). Although gmean $\mathrm{C}_{\text {max,ss }}$ was similar, gmean $\mathrm{AUC}_{\text {ss }}$ was $\sim 20 \%$ lower and gmean $\mathrm{C}_{\text {min,ss }}$ 50\% lower following $200 \mathrm{mg}$ TAB versus $400 \mathrm{mg}$ CAP (Online Resource 1, Supplementary Table 4). Further assessment of steady-state PK was conducted during stage 2; gmean $\mathrm{C}_{\text {max,ss, }}, \mathrm{C}_{\text {min,ss }}$ and $\mathrm{AUC}_{0-12, \mathrm{ss}}$ with 300 and $400 \mathrm{mg}$ TAB $\mathrm{BD}$ in the expansion cohorts exceeded those achieved with the $400 \mathrm{mg}$ BD CAP dose (Table 2).

\subsection{Safety and tolerability}

Tables 3 (dose-expansion and dose-escalation cohorts) and 4 (expansion phase, alternative dosing schedule cohorts) list the median exposure to olaparib, the most commonly reported AEs (Table 4 only) and $\mathrm{G} \geq 3$ AEs. Online Resource 1, Supplementary Table 5 details the most commonly reported AEs for the dose-expansion and dose-escalation cohorts, and Online Resource 1, Supplementary Table 6 summarizes haemoglobin toxicity for groups 6 and 8 .

During TAB dose escalation, the $450 \mathrm{mg}$ BD dose level was deemed non-tolerable; G3 anaemia, thrombocytopenia and neutropenia occurred in 3/6 (50\%), 1/6 (17\%) and 1/6 patients (17\%), respectively; overall, $5 / 6$ patients $(83 \%)$ required a dose reduction because of drug-related AEs. The $400 \mathrm{mg}$ BD TAB dose was defined as the MTD for additional expansion cohorts.

Nausea and vomiting were the most commonly reported AEs in the dose-expansion and dose-escalation phases (reported in $84 \%$ [nausea] and $80 \%$ [vomiting] of patients in groups 1 and $3-6)$; in these groups, $8 \%$ and $1 \%$ of patients had a dose reduction because of nausea and vomiting, respectively. The incidence of G3-4 anaemia and 
thrombocytopenia was higher in the $400 \mathrm{mg} \mathrm{BD}$ group than the $300 \mathrm{mg} \mathrm{BD}$ group (30\% vs $22 \%$ and $18 \%$ vs $0 \%$, respectively).

In 18 patients randomized to the approved $400 \mathrm{mg}$ BD CAP regimen (group 6), anaemia was reported for 6/18 patients (33\%; including four cases of G $\geq 3$ anaemia [22\%]; G1-2 nausea occurred in $15 / 18$ cases [83\%] and vomiting in 5/18 patients [28\%], including one G3 [5.6\%]).

Eight patients in the dose-expansion phase required permanent discontinuation of olaparib because of an AE across dose levels ( $n=1$ patient each: nausea; vomiting; rash; increased AST; increased ALT; small intestinal obstruction; myalgia and rash [both AEs in one patient]; large intestinal obstruction and vomiting [both AEs in one patient]). There were seven deaths altogether; all were related to the disease under investigation. Three of these deaths had an intercurrent serious $\mathrm{AE}$ ( $n=1$ patient each: pneumocystis carinii pneumonia; intra-abdominal haemorrhage; small intestinal obstruction). The $A E$ of pneumocystis carinii pneumonia was thought to be secondary to bone pancytopenia and was considered by the investigator as related to olaparib.

When considering all patients who received 300 or $400 \mathrm{mg}$ BD TAB (during doseescalation and randomized expansion phases), $7 / 24$ (29\% [22\% in randomized expansion phase]) and 14/23 (61\% [65\% in randomized expansion phase]) patients required $\geq 1$ dose reduction because of $A E s$ (mainly due to gastrointestinal toxicities, fatigue, anaemia), respectively. Six patients who started at $400 \mathrm{mg}$ TAB BD required $\geq 2$ dose de-escalations; dose reductions were more common during the first two cycles of therapy compared with other doses and CAP. Online Resource 1, Supplementary Table 7 summarizes drug interruptions and dose reductions for groups 6 and 8. Median time on treatment for ovarian cancer patients receiving $400 \mathrm{mg}$ TAB BD was 212 days, 
although the median duration for patients receiving the cohort starting dose of $400 \mathrm{mg}$ TAB BD before requiring a dose modification was only 51 days.

Intermittent dosing (group 8) did not affect patient adherence to treatment; mean compliance was $94.6-97.4 \%$ across schedules. For the alternative-scheduling cohorts (group 8), the inclusion criteria were amended to restrict enrolment for patients with higher baseline haemoglobin (Online Resource 1, Supplementary Table 1), resulting in a lower incidence of anaemia overall (12 cases, 19.4\%) relative to the patients in the previous expansion cohorts (groups 1 and 6; 22/64, 34.4\%). Three and two cases of G3 anaemia were observed in the intermittent and continuous-dosing groups $(3 / 31,9.6 \%$; 2/31, 6.4\%; Table 4), respectively.

The most common AEs observed in group 8 were nausea and vomiting (reported in $82 \%$ and $68 \%$ of patients, respectively; Table 4). Intermittent administration of $400 \mathrm{mg}$ BD led to G3 vomiting in $18.8 \%$ of cases ( $3 / 16$ patients), while only one case was reported in each of the other dose schedules (6-7\%). Moreover, the $400 \mathrm{mg} \mathrm{BD}$ intermittent dose level had the most patients requiring dose reductions $(6 / 16,37.5 \%)$ because of $A E s$, compared with $6.7-18.8 \%$ of patients in the other dose schedules.

\subsection{Antitumour activity in gBRCAm carriers and serous ovarian carcinoma}

The radiological ORR in $\mathrm{g} B R C A \mathrm{~m}$ carriers with serous ovarian carcinoma from groups 1 and 6 ( $n=53$ total: 200 mg BD TAB, $n=8 ; 300$ mg BD TAB, $n=13 ; 400$ mg BD TAB, $n=12$; $400 \mathrm{mg}$ BD CAP, $n=20)$ was $30 \%(16 / 53 ; 95 \%$ Cl 18.3-44.3) across cohorts, although it appeared higher for patients receiving 300 mg TAB BD (5/13, 38\%; 95\% Cl 13.9-68.4) and $400 \mathrm{mg}$ TAB BD (5/12, 42\%; 95\% Cl 15.2-72.3). The RR based on a RECIST response and/or CA-125 was $40 \%(21 / 53,95 \% \mathrm{Cl} 26.5-54.0)$. 
Analysis of the percentage change in tumour size after 8 and 16 weeks of treatment showed similar efficacy for both 300 and $400 \mathrm{mg}$ TAB compared with $400 \mathrm{mg}$ BD CAP. The one-sided upper limit of the $80 \% \mathrm{Cl}$ was below the pre-specified criteria of $20 \%$ compared with the original formulation (400 mg BD CAP) for both groups at $300 \mathrm{mg}$ BD TAB (LSmean change in tumour size at week 8 : 1.8\%, one-sided $80 \%$ UCL 12.1) and $400 \mathrm{mg}$ BD TAB (LSmean change in tumour size at week 8: $-10.5 \%$, one-sided $80 \%$ UCL 0), indicating similar efficacy. However, the $200 \mathrm{mg}$ BD CAP dose failed to meet the criteria to be considered similar to $400 \mathrm{mg}$ BD CAP (difference in LSmeans at week 8: $8.6 \%$, one-sided $80 \%$ UCL 21.1 ). Consistent results were obtained after 16 weeks of therapy (Tables 5 and 6, Online Resource 1, Supplementary Figure 2). For patients in the alternative-scheduling cohorts (group 8), only $200 \mathrm{mg}$ TID continuous and $400 \mathrm{mg}$ BD intermittent dose schedules were considered non-inferior to $400 \mathrm{mg}$ BD CAP (Table 6, Online Resource 1, Supplementary Figure 2). 


\section{DISCUSSION}

This adaptive trial was planned as a comparative bioavailability study to select the optimal dose of a TAB olaparib formulation so as to simplify drug administration. Prior clinical trials using the CAP olaparib formulation reported a direct association between dose and antitumour activity in gBRCAm carriers [8-10]. We conducted a comparison of olaparib TAB bioavailability, efficacy, and safety with those of $400 \mathrm{mg}$ CAP BD and identified $300 \mathrm{mg}$ TAB BD as the recommended TAB monotherapy dose for investigation in olaparib Phase III clinical trials.

PK modelling predicted that a $200 \mathrm{mg}$ BD TAB dose would deliver multiple-dose exposure within the range of exposures observed with $400 \mathrm{mg}$ BD CAP [7]. However, direct intra-subject comparison of steady-state exposure for the six patients treated at $200 \mathrm{mg}$ BD TAB showed that, although the gmean $C_{\max , \text { ss }}$ achieved was similar to 400 mg BD CAP, gmean $\mathrm{AUC}_{\mathrm{ss}}$ and $\mathrm{C}_{\mathrm{min}, \mathrm{ss}}$ were approximately $20 \%$ and $50 \%$ lower, respectively. Consequently, stage 2 of the study was pursued to assess tolerability and antitumour activity of higher TAB doses.

The dose-escalation phase established $400 \mathrm{mg} \mathrm{BD}$ TAB as the MTD based on haematological toxicities at $450 \mathrm{mg} \mathrm{BD}$. However, as patient numbers in this phase were small, a randomized comparison between $300 \mathrm{mg}$ BD TAB (lowest dose achieving similar drug exposure to the CAP recommended dose), $400 \mathrm{mg}$ BD TAB (the defined TAB MTD) and the original $400 \mathrm{mg}$ CAP regimen was performed to better determine the tolerability profiles. For patients who were receiving $400 \mathrm{mg} \mathrm{BD}$ TAB in the randomized dose-expansion phase, the number of anaemia and gastrointestinal AEs, as well as the number of patients requiring dose reductions, confirmed that olaparib $400 \mathrm{mg}$ BD TAB was not optimally tolerated in this population of heavily pre-treated breast and ovarian cancer patients. 
Although antitumour activity was not a primary objective of this study and there were limited numbers of patients in each dose subgroup, it is noteworthy that this is one of the largest series of $\mathrm{gBRCAm}$ carriers receiving a single-agent PARP inhibitor. The overall response rate across the different cohorts was $30 \%$ in gBRCAm ovarian cancer patients; these data further support the confirmed anticancer activity of olaparib in patients with a gBRCAm [11, 17]. For ovarian cancer patients, the analysis of preliminary data on antitumour activity showed that $300 \mathrm{mg} \mathrm{BD}$ TAB was similar to the approved dose of 400 mg BD CAP.

We also evaluated olaparib administered in different schedules based on the hypothesis that different patterns of drug exposure may help alleviate common AEs of anaemia, nausea or vomiting. Intermittent PARP inhibition has been investigated in several combinatory trials, mainly with cytotoxic chemotherapy [24-26], but this is, to our knowledge, the first series of patients prospectively enrolled to receive a single-agent PARP inhibitor in different intermittent schedules. In these cohorts, intermittent scheduling did not improve gastrointestinal tolerability; indeed, nausea, vomiting or diarrhoea seemed to be better controlled by reducing the daily amount of drug administered. However, this cohort was conducted only in patients with advanced ovarian carcinoma, where serosal bowel involvement is a common cause of gastrointestinal symptoms, and may not be generalizable to other tumour types. Additionally, preclinical data have indicated that continuous inhibition of PARP is required for maximum efficacy (unpublished data), further supporting continuous rather than intermittent dosing for olaparib monotherapy. Administration of the $600 \mathrm{mg}$ total daily dose as a TID schedule (200 mg TID), while showing similar efficacy, was not considered to improve tolerability over $300 \mathrm{mg} \mathrm{BD}$, with additional patient inconvenience of TID dosing. 


\section{CONCLUSION}

In conclusion, this multistage clinical trial showed that patients' exposure following tablet doses $\geq 300 \mathrm{mg}$ BD matched or exceeded that of the approved $400 \mathrm{mg}$ BD CAP (8x50 mg capsules BD). A dose of $300 \mathrm{mg}$ BD TAB was better tolerated than higher doses, with similar clinical activity in terms of tumour shrinkage. None of the intermittent-dosing schedules explored improved both efficacy and tolerability relative to $300 \mathrm{mg} \mathrm{BD}$ continuous dosing. Therefore, continuous dosing of olaparib tablets $300 \mathrm{mg} \mathrm{BD}(2 \times 150$ mg tablets $\mathrm{BD}$ ) is recommended for olaparib Phase III clinical trials, simplifying drug administration from 16 capsules to four tablets per day. 


\section{COMPLIANCE WITH ETHICAL STANDARDS}

All procedures performed in studies involving human participants were in accordance with the ethical standards of the institutional and/or national research committee and with the 1964 Helsinki declaration and its later amendments or comparable ethical standards. Informed consent was obtained from all individual participants included in the study.

\subsection{Funding}

This study was sponsored by AstraZeneca. Medical writing assistance was provided by Claire Routley PhD from Mudskipper Business Ltd, funded by AstraZeneca.

\subsection{Disclosure of potential conflicts of interest}

JM, VM, AG, CS and KL have no conflicts. SBK has attended Advisory Boards for AstraZeneca and has assisted AstraZeneca with the EMA regulatory submission for olaparib. ED received commercial funding from AstraZeneca to The Christie NHS Foundation Trust for the conduct of this study. MM reports grants from Cancer Research UK during the conduct of this study; grants from Roche, AstraZeneca, GSK; personal fees from Amgen, Roche, GlaxoSmithKline, Eisai, Bristol-Myers Squibb; study fees from Novartis, Astellas (was OSI), Millenium, Abbott (now AbbVie), Clovis Oncology, Pfizer, Bristol-Myers Squibb, Vertex, Eisai, Merck, Immunocore; Advisory Board attendance from Bristol-Myers Squibb; and travel funding from Merck and Immunocore. MF has received provision of drugs for investigator-initiated trials and Advisory Board attendance from AstraZeneca. CG has received personal and non-personal fees from AstraZeneca for Advisory Board attendance, lectures and research funding. RP has received clinical trial funding and Advisory Board payment from AstraZeneca. GR has received funding for Advisory Board attendance from AstraZeneca. SN has received funding for Advisory 
Board attendance from AstraZeneca and Roche. JL has received personal and nonpersonal fees from AstraZeneca for Advisory Board attendance and lectures. HS was previously employed by AstraZeneca and owns stock in AstraZeneca. AF is employed by and owns stock in AstraZeneca. WB is a contract researcher for AstraZeneca. RM reports commercial funding for the conduct of this study from AstraZeneca to The Royal Marsden/Institute of Cancer Research. 


\section{REFERENCES}

1. Farmer H, McCabe N, Lord CJ et al (2005) Targeting the DNA repair defect in BRCA mutant cells as a therapeutic strategy. Nature 434:917-921

2. Bryant HE, Schultz N, Thomas HD et al (2005) Specific killing of BRCA2-deficient tumours with inhibitors of poly(ADP-ribose) polymerase. Nature 434:913-917

3. Pal T, Permuth-Wey J, Betts JA et al (2005) BRCA1 and BRCA2 mutations account for a large proportion of ovarian carcinoma cases. Cancer 104:2807-2816

4. Cancer Genome Atlas Research Network (2011) Integrated genomic analyses of ovarian carcinoma. Nature 474:609-615

5. Gonzalez-Angulo AM, Timms KM, Liu S et al (2011) Incidence and outcome of BRCA mutations in unselected patients with triple receptor-negative breast cancer. Clin Cancer Res 17:1082-1089

6. Hartman AR, Kaldate RR, Sailer LM et al (2012) Prevalence of BRCA mutations in an unselected population of triple-negative breast cancer. Cancer 118:2787-2795

7. Fong PC, Boss DS, Yap TA et al (2009) Inhibition of poly(ADP-ribose) polymerase in tumors from BRCA mutation carriers. N Engl J Med 361:123-134

8. Audeh MW, Carmichael J, Penson RT et al (2010) Oral poly(ADP-ribose) polymerase inhibitor olaparib in patients with $B R C A 1$ or $B R C A 2$ mutations and recurrent ovarian cancer: a proof-of-concept trial. Lancet 376:245-251 
9. Tutt A, Robson M, Garber JE et al (2010) Oral poly(ADP-ribose) polymerase inhibitor olaparib in patients with $B R C A 1$ or $B R C A 2$ mutations and advanced breast cancer: a proof-of-concept trial. Lancet 376:235-244

10. Kaye SB, Lubinski J, Matulonis U et al (2012) Phase II, open-label, randomized, multicenter study comparing the efficacy and safety of olaparib, a poly (ADP-ribose) polymerase inhibitor, and pegylated liposomal doxorubicin in patients with BRCA1 or BRCA2 mutations and recurrent ovarian cancer. J Clin Oncol 30:372-379

11. Kaufman B, Shapira-Frommer R, Schmutzler RK et al (2015) Olaparib monotherapy in patients with advanced cancer and a germ-line BRCA1/2 mutation. J Clin Oncol $33: 244-250$

12. Gelmon KA, Tischkowitz M, Mackay $\mathrm{H}$ et al (2011) Olaparib in patients with recurrent high-grade serous or poorly differentiated ovarian carcinoma or triple-negative breast cancer: a phase 2, multicentre, open-label, non-randomised study. Lancet Oncol $12: 852-861$

13. Konstantinopoulos PA, Spentzos D, Karlan BY et al (2010) Gene expression profile of BRCAness that correlates with responsiveness to chemotherapy and with outcome in patients with epithelial ovarian cancer. J Clin Oncol 28:3555-3561

14. Michels J, Vitale I, Saparbaev M et al (2014) Predictive biomarkers for cancer therapy with PARP inhibitors. Oncogene 33:3894-3907

15. McCabe N, Turner NC, Lord CJ et al (2006) Deficiency in the repair of DNA damage by homologous recombination and sensitivity to poly(ADP-ribose) polymerase inhibition. Cancer Res 66:8109-8115 
16. Ledermann J, Harter P, Gourley C et al (2012) Olaparib maintenance therapy in platinum-sensitive relapsed ovarian cancer. N Engl J Med 366:1382-1392

17. Ledermann J, Harter P, Gourley C et al (2014) Olaparib maintenance therapy in patients with platinum-sensitive relapsed serous ovarian cancer: a preplanned retrospective analysis of outcomes by BRCA status in a randomised phase 2 trial. Lancet Oncol 15:852-861

18. AstraZeneca. Lynparza ${ }^{\mathrm{TM}}$ approved in the European Union as first-in-class treatment for advanced BRCA-mutated ovarian cancer. 2014. Available at: http://www.astrazeneca.com/Media/Press-releases/Article/20141218--lynparzaapproved-in-the-european-union.

19. FDA. Olaparib. 2014. Available at: http://www.fda.gov/Drugs/InformationOnDrugs/ApprovedDrugs/ucm427598.htm.

20. AstraZeneca. Global Policy: Bioethics. 2015. Available at: http://www.astrazeneca.com/Responsibility/Code-policies-standards/Our-globalpolicies.

21. Therasse P, Arbuck SG, Eisenhauer EA et al (2000) New guidelines to evaluate the response to treatment in solid tumors. European Organization for Research and Treatment of Cancer, National Cancer Institute of the United States, National Cancer Institute of Canada. J Natl Cancer Inst 92:205-216

22. Rustin GJ, Vergote I, Eisenhauer E et al (2011) Definitions for response and progression in ovarian cancer clinical trials incorporating RECIST 1.1 and CA 125 
agreed by the Gynecological Cancer Intergroup (GCIG). Int J Gynecol Cancer 21:419423

23. Hennessy BT, Coleman RL, and Markman M (2009) Ovarian cancer. Lancet 374:13711382

24. Balmana J, Tung NM, Isakoff SJ et al (2014) Phase I trial of olaparib in combination with cisplatin for the treatment of patients with advanced breast, ovarian and other solid tumors. Ann Oncol 25:1656-1663

25. Del Conte G, Sessa C, von Moos R et al (2014) Phase I study of olaparib in combination with liposomal doxorubicin in patients with advanced solid tumours. $\mathrm{Br} \mathrm{J}$ Cancer 111:651-659

26. Oza AM, Cibula D, Benzaquen AO et al (2015) Olaparib combined with chemotherapy for recurrent platinum-sensitive ovarian cancer: a randomised phase 2 trial. Lancet Oncol 16:87-97 


\section{FIGURE LEGENDS}

Fig 1 Mean olaparib plasma concentration profile for (a) single oral dosing of the CAP formulation at doses of 50,100 and $400 \mathrm{mg}$ to patients with advanced solid tumours, (b) following single oral dosing of the TAB formulation at doses of 25,50 and $250 \mathrm{mg}$ to patients with advanced solid tumours, and (c) following multiple dosing of the TAB (200 $\mathrm{mg} \mathrm{BD}$ ) and CAP (400 $\mathrm{mg} \mathrm{BD}$ ) to patients in group 